\title{
Warning signal as a component of a compound stimulus in human eyelid conditioning
}

JONATHAN BERMAN, CARL D. WILLIAMS AND NEIL SCHNEIDERMAN UNIVERSITY OF MIAMI

One hundred human $S$ s conditioned and extinguished in a parallel fashion to both the warning signal of a masking probability learning task and the programmed CS. Acquisition and extinction curves were similar for early and nonearly responders though early responders had a higher response rate during acquisition.

Even though Ss have been given as many as 50 to 80 acquisition trials in human eyelid conditioning, extinction typically requires only a very few trials. In contrast, extinction of the conditioned eyelid response in animals is relatively slow. In an attempt to account for the differential extinction rate between humans and animals, Spence (1966a) suggested that the human $S$ readily discriminates extinction from acquisition because the US is discontinued during extinction. Once this discrimination is made, $\mathrm{S}$ rapidly develops a set not to respond to the CS so that the rapid extinction in humans results from a sudden decrease in drive level. Thus, according to Spence, extinction data from traditional eyelid conditioning studies in humans do not accurately portray the extinction of a learned process.

Spence and associates have embedded conditioning and extinction in the context of a masking probability learning task in an effort to prevent Ss from discriminating between acquisition and extinction. The masking situation involves instructing $S s$ that the study is an investigation of the effects of distraction on performance, that the task is to learn to predict which of two lateral lights will come on following the illumination of a centrally-placed warning light, and that a tone and an air puff to the eye will be given as distracting stimuli. Striking increases in resistance to extinction have been observed when the masking situation is employed. Spence (1966b), for example, found the extinction curve to be above the spontaneous blinking rate even after 50 extinction trials.

An alternative to Spence's inhibitory set hypothesis is that the masking procedure provides a compound stimulus so that Ss develop greater resistance to extinction by becoming conditioned to more than one stimulus element. The present experiment compared CRs to the warning signal with CRs to the traditional CS over both acquisition and extinction.

\section{Method}

Each of 109 Ss received three preconditioning trials followed by 21 acquisition and 24 extinction trials. Intertrial intervals of 15,25 , and $35 \mathrm{sec}$ were presented in random order with a mean of $25 \mathrm{sec}$. Nine
Ss failed to meet the conditioning criterion of four CRs during acquisition. The US was a $2 \mathrm{~mA} 100 \mathrm{msec}$ duration infraorbital shock administered $500 \mathrm{msec}$ after the onset of the tone CS. The CS and US offset simultaneously. Eyelid closures of at least $1 \mathrm{~mm}$ deflection from the baseline in the interval from 200$500 \mathrm{msec}$ after $\mathrm{CS}$ onset in acquisition and from 200$1200 \mathrm{msec}$ after $\mathrm{CS}$ onset in extinction were considered as CRs. Two Ss, separated by a partition, viewed three 25-W light bulbs located across the room. The center bulb was red and the outside two were blue. The red warning light of 1 sec duration preceded each onset of the tone by $1 \mathrm{sec}$. One of the two side lights immediately followed the offset of the tone and shock and remained on for $1 \mathrm{sec}$. In each sequence of 10 trials, seven of the side lightings were to S's right and three to his left. The $\mathrm{S}$ was instructed that when the red light appeared he was to indicate his guess as to which of the two blue lights would be correct by moving a two-way switch mounted in front of him. In extinction, the warning signal, the tone, and the blue light were presented but the shock was omitted. Results

Acquisition and extinction curves for $100 \mathrm{Ss}, 46$ males and 54 females, to the warning signal (red light) and to the tone are presented in Fig. 1. After acquisition Trial 12, there was slightly more responding to the tone than to the light. Analyses of variance indicated that reliable conditioning $(p<.001)$ occurred to both the warning signal and the tone, but that the differences in level of conditioning between signals were not significant. In extinction Ss responded significantly more to the tone than to the warning signal

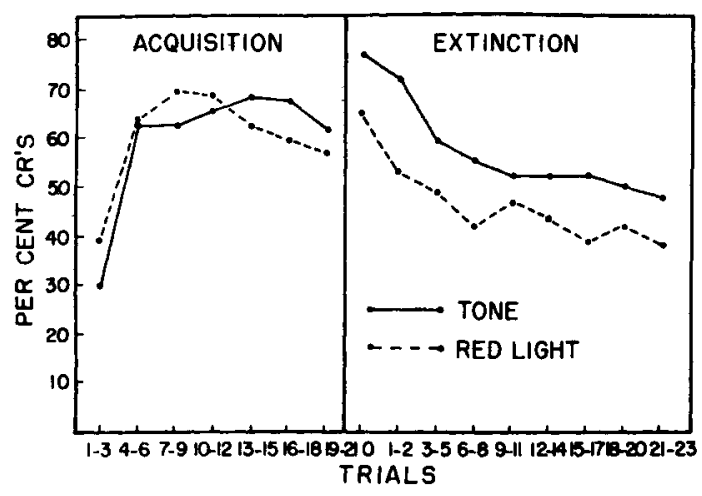

Fig. 1. Percentage of warning signal CRs and tone CRs during acquisition and extinction. 


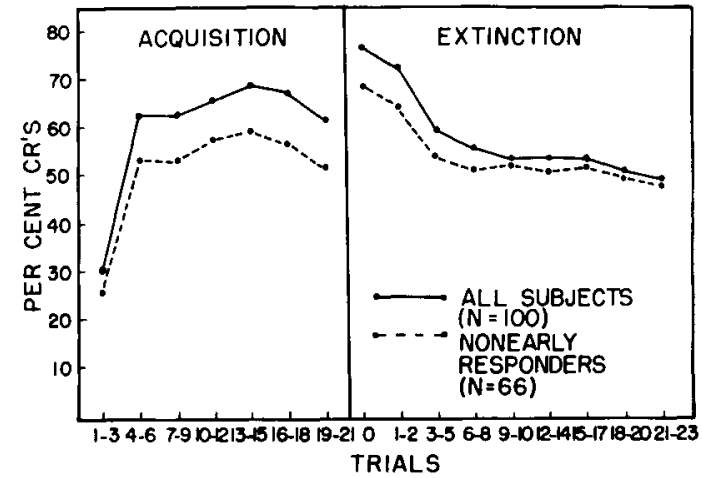

Fig. 2. Percentage of tone CRs for all Ss and for nonearly responders during acquisition and extinction.

$(\mathrm{p}<.007)$, although resistance to extinction was considerable to both stimuli.

In the present study, Ss were classified as early responders to the tone using Spence and Taylor's latency criterion for "voluntary responders." As may be seen in Fig. 2, elimination of 34 of the 100 Ss as early responders lowered the level of responding more during acquisition than in extinction but did not influence the shape of the curves. Analyses of variance conducted on the means of the blocks for all Ss and for the nonearly responders indicated that the trends of the curves did not differ in acquisition or in extinction. The differences between the nonearly and all responders were reliable $(p<.005)$ in acquisition but not in extinction.

\section{Discussion}

The major finding of the present experiment was that responses to the warning signal were acquired and extinguished in a parallel fashion to responses to the tone. The fact that Ss became conditioned to more than one stimulus element suggests that other theories, such as statistical learning theory, may be able to account for the increased resistance to extinction observed in the masking situation as efficiently as Spence's hypothesized elimination of a set not to respond based on S's discrimination of the stimulus change at the beginning of extinction. In order to mask the purpose of the experiment from his Ss, Spence concurrently introduced several innovations into his procedures. These included (a) a unique set of instructions, (b) the guessing task itself, and (c) pairing of a warning signal with shock during half of his trials in extinction. Since the warning signal was paired with tone as well as with shock during acquisition training, possible stimulus compounding effects may have altered the interstimulus interval function ordinarily relating the temporal relationship of a warning signal and shock. In any event, before Spence's explanation can be accepted, the effects of instructions, the guessing task, and warning signal-shock pairings in extinction have to be evaluated. Such an assessment is currently being conducted in our laboratory. The higher level of responding to the tone than to the warning signal during extinction and after Trial 12 during acquisition apparently reflected the greater contiguity of the tone than the warning signal with the shock.

The present finding of reliably more CRs to the tone during acquisition by early than nonearly responders is consistent with the hypothesis that early responders represent a class of Ss with a lower threshold for noxious stimuli whose early blinking leads to higher conditioning rates through facilitation of the establishment of a CR-US overlap. A given US level should be functionally more intense for Ss with a low pain threshold than for other Ss. Since response latency decreases as US intensity increases (Gormezano \& Moore, 1962), low pain threshold Ss should respond more quickly than other Ss. Therefore, such Ss should establish a CR-US overlap sooner which should be manifested in higher conditioning rates. The unusually high percentage of early responders in the present study, perhaps a function of the use of infraorbital shock, is also consistent with the low pain-threshold hypothesis if the $2 \mathrm{~mA}$ shock employed in the present study is more aversive than an air puff of the intensity typically used in studies that have identified "voluntary responders." The above analysis and the present finding of similar acquisition and extinction curves for early and nonearly responders suggests that the elimination of Ss on the basis of a latency criterion is unwarranted.

\section{References}

GORMEZANO, I., \& MOORE, J. W. Effects of instructional set and UCS intensity on the latency, percentage, and form of the eyelid response. J. exp. Psychol., 1962, 63, 487-494.

SPENCE, K. W. Cognitive and drive factors in the extinction of the conditioned eye blink in human subjects. Psychol. Rev., 1966a, 73, 445-458.

SPENCE, K. W. Extinction of the human eyelid CR as a function of presence or absence of the UCS during extinction. J. exp. Psychol., $1966 \mathrm{~b}, 71,642-648$.

Note

1. Supported by NASA grant NGR 10-007-010. 